\title{
The long non-coding RNA LINC00460 predicts the prognosis and promotes the proliferation and migration of cells in bladder urothelial carcinoma
}

\author{
LIJIE WEN ${ }^{1}$, XILING ZHANG ${ }^{1}$, JING BIAN ${ }^{2}$, LI HAN ${ }^{2}$, HAIBO HUANG ${ }^{3}$, \\ MIAO $\mathrm{HE}^{2}$, MINJIE WEI ${ }^{2}$ and PING WANG ${ }^{1}$ \\ ${ }^{1}$ Department of Urology, The Fourth Affiliated Hospital of China Medical University, Shenyang, Liaoning 110032; \\ ${ }^{2}$ School of Pharmacy, Department of Pharmacology, China Medical University, Shenyang, Liaoning 110122; \\ ${ }^{3}$ Department of Surgical Oncology and General Surgery, The First Hospital of China Medical University, Shenyang, \\ Liaoning 110001, P.R. China
}

Received January 23, 2018; Accepted January 24, 2019

DOI: $10.3892 / 01.2019 .10023$

\begin{abstract}
Long non-coding RNAs (lncRNAs) may serve an important role in cancer development and may also be suitable for use as prognostic biomarkers. At present, the role of lncRNAs in bladder cancer remains unclear. The present study examined the potential involvement of IncRNA LINC00460 in bladder urothelial carcinoma using data from The Caner Genome Atlas (TCGA) and cell line experiments. The results indicated that LINC00460 expression levels were increased in bladder urothelial carcinoma tissues and bladder cancer 5637 and T24 cell lines compared with corresponding normal controls $(\mathrm{P}<0.05)$. TCGA data indicated that LINC00460 expression was negatively correlated with a positive prognosis in patients with bladder urothelial carcinoma $(\mathrm{P}<0.05)$. Consistently, the downregulation of LINC00460 with short hairpin RNA significantly suppressed 5637 and T24 cell proliferation and migration. Therefore, it was suggested that strategies that target LINC00460 may be developed as novel therapeutic approaches for the treatment of bladder cancer. In addition, the expression level of androgen receptor (AR) was downregulated in bladder urothelial carcinoma tissues and exhibited a negative correlation with the expression level of LINC00460 ( $\mathrm{r}=-0.43 ; \mathrm{P}<0.0001)$, based on the data from TCGA. We hypothesized that LINC00460 may serve an oncogenic role by regulating the expression of AR.
\end{abstract}

Correspondence to: Dr Ping Wang, Department of Urology, The Fourth Affiliated Hospital of China Medical University, 4 Chongshandong Road, Shenyang, Liaoning 110032, P.R. China E-mail: cmu4hwlj@126.com

Key words: bladder cancer, long non-coding RNA, prognosis

\section{Introduction}

Bladder cancer is the seventh most common type of cancer in the male population worldwide, and eleventh including females (1). There are 2 major types of bladder cancer: Non-muscle invasive bladder cancer (NMIBC) and muscle-invasive bladder cancer (MIBC). Bladder cancer exhibits a high frequency of relapse and a poor clinical outcome once the tumors progress to muscle-invasive disease (2). Patients with MIBC have a poor prognosis due to the aggressive nature of the tumor and its resistance to chemo- and radiotherapy (3). Furthermore, for patients with MIBC, the risk of developing lymph node metastases is increased and chemotherapy is less effective in comparison with patients with NMIBC (4). Therapies and prognosis for this type of cancer depend on the clinical information and individual tumor pathology. However, in certain cases, even when tumors present with similar histology, they respond differently to the same treatment, resulting in different survival outcomes for the patients (5).

Protein-coding genes constitute only $2 \%$ of the total genome sequence; the remaining sequences produce various classes of functional non-coding RNAs (6). There are two major classes of non-coding RNAs, including small and long non-coding RNAs (lncRNAs). The difference between lncRNAs and small non-coding RNAs is primarily their size, with the length of lncRNAs defined as being >200 nucleotides. Similar to other non-coding RNAs, lncRNA transcripts are associated with a wide range of cancer types, including bladder cancer (7-10). Alterations in the expression levels of lncRNAs have been demonstrated to be associated with a number of important cellular functions, and may promote the migration and invasion of cancer cells $(4,11)$. Non-coding RNAs have attracted increasing attention in previous years regarding their role in bladder cancer, with the number of studies on this topic increasing considerably. The combination of traditional methods of treatment with the use of non-coding RNAs may provide improved therapy for patients. The accumulation of data in this field may assist in elucidating the molecular profiles of patients with bladder cancer and contribute to the 
use of non-coding RNAs as tools for precision medicine to target critical genes in bladder cancer.

LINC00460 is a human IncRNA gene, transcribed from chromosome 13, measuring $935 \mathrm{bp}$; its function is poorly understood. Recent studies have demonstrated that LINC00460 is associated with kidney cancer, nasopharyngeal carcinoma and head and neck squamous cell carcinoma (12-14). A previous study demonstrated, using a bioinformatics approach, that LINC00460 may serve an important role in tumorigenesis and metastasis through the regulation of the cell cycle and cell death (15). During the analysis of the differential expression of lncRNAs in bladder cancer tissues in the present study, it was identified that LINC00460 was highly upregulated compared with the normal adjacent tissue. Furthermore, the upregulation of LINC00460 was demonstrated to be associated with the poor survival of patients. We hypothesized that this lncRNA may serve an important role in the regulation of biological processes in bladder urothelial carcinoma. However, the precise function and the underlying molecular mechanisms remain to be elucidated.

Bladder cancer predominantly presents in men (16). Factors that are exclusive to males are likely to serve critical roles in the development of bladder cancer $(17,18)$. An increasing amount of evidence has suggested the involvement of androgen receptor (AR) signaling in the development and progression of bladder cancer (19-21). The present study investigated the role of the lncRNA LINC00460 in bladder urothelial carcinoma using The Cancer Genome Atlas (TCGA) data and cell experiments. The results indicated that increased LINC00460 expression was a characteristic molecular change in bladder urothelial carcinoma tissues, and in 5637 and T24 cell lines. Therefore, the effects of aberrant LINC00460 expression on the biological behavior of 5637 and T24 cells were additionally investigated. The results provided novel insights into the function and mechanisms of LINC00460 in bladder urothelial carcinoma pathogenesis, and identified LINC0046 as a potential therapeutic target for cancer intervention.

\section{Materials and methods}

TCGA database. Gene expression data obtained by RNA sequencing and the corresponding clinical data for 412 patients (including 413 samples) with bladder urothelial carcinoma were downloaded from TCGA (https://cancergenome.nih.gov/). All RNA expression levels of the samples were normalized. The edgeR Bioconductor package was used to analyze P-values and fold-change (FC) using R (version 3.4.0) (22). A gene was defined as differentially expressed between cancerous and normal tissues when the false discovery rate-adjusted $\mathrm{P}<0.01$ and the FC was $\geq 2$-fold increased or decreased.

Cell culture and transfection. The expression of LINC00460 and the basic characterization of bladder urothelial carcinoma cell lines were initially investigated using the Expression Atlas database (23) and the American Type Culture Collection website (https://www.atcc.org/), respectively (Table I). Based on these results, the 5637 cell line was selected, as the expression of LINC00460 was the highest in these cells compared with the others included in the analysis. From the verification of LINC00460 expression using reverse transcription-quantitative polymerase chain reaction (RT-qPCR) in bladder cancer cell lines (T24, J82, TCCSUP and UM-UC-3), it was identified that LINC00460 was also upregulated in the T24 cell line compared with the normal bladder epithelial SV-HUC-1 cell line (data not show).

The 5637, T24, J82, TCCSUP, UM-UC-3 and SV-HUC-1 cells were purchased from The Cell Bank of Type Culture Collection of Chinese Academy of Sciences (Shanghai, China). The 5637 and T24 cells were cultured in RPMI-1640 medium (Gibco; Thermo Fisher Scientific, Inc., Waltham, MA, USA) and the SV-HUC-1 cell line was cultured in F12 K medium (Gibco; Thermo Fisher Scientific, Inc.). J82, TCCSUP and UM-UC-3 cells were cultured in Dulbecco's modified Eagle's medium (Gibco; Thermo Fisher Scientific, Inc.). These media were supplemented with $10 \%$ fetal bovine serum (Gibco, Thermo Fisher Scientific, Inc.) and 1\% penicillin and streptomycin, in a humidified atmosphere with $5 \% \mathrm{CO}_{2}$ at $37^{\circ} \mathrm{C}$.

A total of three short hairpin RNAs (shRNAs) targeting LINC00460 were purchased from Shanghai GenePharma Co., Ltd. (Shanghai, China) and transfected into 5637 and T24 cells with Lipofectamine ${ }^{\circledR} 3000$ (Invitrogen; Thermo Fisher Scientific, Inc.) according to the manufacturer's protocol. The three shRNA sequences specially targeting LINC00460 were designed and cloned into a pGU6/green fluorescent protein (GFP)/Neo-shRNA vector (Shanghai GenePharma Co., Ltd.). Cells (1x105 T24 cells/well; 3x105 5,637 cells/well) were seeded in six-well culture plates and transfected with the $2.5 \mu \mathrm{g}$ sh-LINC00460 in each well. The most effective shRNA sequence (sh-3) in achieving knockdown of LINC00460 expression was selected for subsequent experiments $48 \mathrm{~h}$ after the transfection. The sh-LINC00460 sequences were as follows: sh-1, 5'-GCTAAGACCTAATAGCCAATA-3'; sh-2, 5'-GCC ATCCACTTCAAAGTATTC-3'; and sh-3, 5'-ACCTTGGTC AAACGTTTAACC-3'. A scrambled shRNA was used as the negative control (sh-NC) in the experiments with the following sequence: 5'-GTTCTCCGAACGTGTCACGT-3'.

$R T$-qPCR assays. Total RNA was extracted from 5637, T24, J82, TCCSUP, UM-UC-3 and SV-HUC-1 cells using TRIzol ${ }^{\circledR}$ reagent (Thermo Fisher Scientific, Inc.). Reverse transcription was conducted using the PrimeScript ${ }^{\mathrm{TM}} \mathrm{RT}$ reagent kit with gDNA Eraser (Takara Biotechnology Co., Ltd., Dalian, China). qPCR was performed to detect the expression of LINC00460 using SYBR ${ }^{\circledR}$ Premix Ex Taq ${ }^{\mathrm{TM}}$ II kit (Takara Biotechnology Co., Ltd.). RT-qPCR assays were performed using an Applied Biosystems QuantStudio 3 system (Applied Biosystems; Thermo Fisher Scientific, Inc.), with $\beta$-actin as an endogenous control. The primer sequences used were as follows: LINC00460 forward, 5'-CGAGAAGGCCACCTA TGAGC-3' and reverse, 5'-TGAAGTGGATGGCTCAGG AA-3'; $\beta$-actin forward, 5'-CCGTGAAAAGATGACCCAGAT C-3' and reverse, 5'-CACAGCCTGGATGGCTACGT-3'. A two-step qPCR was performed as follows: Initial denaturation in $95^{\circ} \mathrm{C}$ for $30 \mathrm{sec}$; 40 of cycles of denaturation at $95^{\circ} \mathrm{C}$ for $5 \mathrm{sec}$; and annealing and elongation at $60^{\circ} \mathrm{C}$ for $34 \mathrm{sec}$. The $2^{-\Delta \Delta \mathrm{Cq}}$ method was used to quantify LINC00460 (24).

Cell proliferation assay. Cells were seeded in 96-well plates at a density of $1.0 \times 10^{4}$ and $1.5 \times 10^{4}$ cells per well for T24 and 5637 
Table I. Expression of LINC00460 and characterization of bladder cancer cell lines from the Expression Atlas database and the website of American Type Culture Collection cell lines.

\begin{tabular}{lcccl}
\hline Cell line & $\begin{array}{c}\text { Expression value, } \\
\text { transcripts per million }\end{array}$ & Histological grade & Age & Sex \\
\hline 5637 & 51 & Grade 2 & 68 & Male \\
U-BLC1 & 43 & Grade 3 & 84 & Female \\
HT-1197 & 11 & Grade 4 & 44 & Male \\
TCCSUP & 4 & Grade 4 & 67 & Female \\
J82 & 3 & Grade 3 & 58 & Male \\
RT-112 & 0.9 & Grade 2 & Not reported & Female \\
253J & 0 & Grade 4 & 53 & Male \\
HT-1376 & 0 & Grade 3 & 58 & Female \\
RT4 & 0 & Grade 1 & 63 & Male \\
SW780 & 0 & Grade 1 & 80 & Female \\
T24 & 0 & Grade 3 & 81 & Female \\
\hline
\end{tabular}

cells, respectively. Following incubation at $37^{\circ} \mathrm{C}$ with $5 \% \mathrm{CO}_{2}$ for $0,24,48$ and $72 \mathrm{~h}$, the absorbance of each sample was measured at $450 \mathrm{~nm}$. Cell proliferation was evaluated using a Cell Counting Kit-8 (CCK-8) assay (Dojindo Molecular Technologies, Inc., Shanghai, China) according to the manufacturer's protocol.

Cell migration assay. The 5637 and T24 cells were seeded $\left(1 \times 10^{6}\right.$ cells/plate) in 6-well plates and incubated in serum-free RPMI-1640 medium. A wound was produced using a sterile $100 \mu 1$ pipette tip when a confluent cell monolayer had formed. The size of the wound was measured using an inverted light microscope (magnification, x200) and images were captured at 0 and $24 \mathrm{~h}$.

Statistical analysis. SPSS 23.0 software (IBM Corp., Armonk, NY, USA) was used to perform statistical analysis. The differential expression levels of LINC00460 and AR between the cancerous and adjacent tissues were analyzed with t-tests. The differences in LINC00460 expression between bladder cancer 5637 and T24 cell lines and the normal bladder epithelial SV-HUC-1 cell line was assessed using a one-way analysis of variance with Tamhane's post-hoc test. The correlation between LINC00460 and AR was analyzed by Spearman's rank correlation analysis following logarithmic $\left(\log _{10}\right)$ conversion of the original data. For the survival analysis, the LINC00460 expression data matrix and clinical data files were matched for each sample using the sample ID. The samples were divided into the LINC00460-low and LINC00460-high groups based on the cut-off of the median value, and according to sex. The LINC00460-high group contained the samples with an exact median value. A Kaplan-Meier plot was generated using the survival package in $\mathrm{R}$ (version 3.4.0) with log-rank tests. $\mathrm{P}<0.05$ was considered to indicate a statistically significant difference.

\section{Results}

LINC00460 is upregulated in bladder urothelial carcinoma tissues and cell lines. The differences in LINC00460 expression between the bladder urothelial carcinoma tissues and the normal controls were analyzed using TCGA data. LINC00460 was significantly upregulated in bladder urothelial carcinoma tissues compared with the normal controls $(\mathrm{P}<0.0001$; Fig. 1A). The expression level of LINC00460 was detected by RT-qPCR in bladder urothelial carcinoma and normal bladder epithelial cell lines. An increase in LINC00460 expression was observed in bladder cancer 5637 and T24 cell lines compared with the normal bladder epithelial SV-HUC-1 cell line ( $\mathrm{P}<0.05$; Fig. 1B).

Upregulation of LINC00460 is associated with poor survival. LINC00460 expression levels and the clinical data of 412 patients with bladder urothelial carcinoma were downloaded from TCGA. All patients, male and female, were divided into LINC00460-high and LINC00460-low groups using the mean expression value as the cut-off. The survival time was plotted using a Kaplan-Meier curve stratified by LINC00460-high and LINC00460-low groups. High levels of LINC00460 were significantly associated with a decreased survival time in male patients ( $\mathrm{P}=0.03229$; Fig. $2 \mathrm{~A})$. There was a similar association observed in the overall group ( $\mathrm{P}=0.04678$; Fig. 2B), but not among the female patients ( $\mathrm{P}=0.42445$; Fig. 2C).

Downregulation of LINC00460 inhibits the proliferation and migration of bladder urothelial carcinoma cells. To explore the function of LINC00460, an shRNA targeting LINC00460 was transfected into 5637 and T24 cells. GFP visualization indicated that the shRNA was efficiently transfected into cells (Fig. 3A). RT-qPCR indicated that LINC00460 was significantly downregulated at $48 \mathrm{~h}$ after transfection of shRNA in the 5637 and T24 cell lines compared with the control group ( $\mathrm{P}<0.05$; Fig. 3B). A CCK-8 assay demonstrated that the downregulation of LINC00460 inhibited the proliferation of 5637 and $\mathrm{T} 24$ cells in vitro $(\mathrm{P}<0.05$; Fig. $3 \mathrm{C})$. In addition, the effect of LINC00460 on the migration capacity of 5637 and T24 cells was observed via a wound-healing assay. The wound-healing 
A

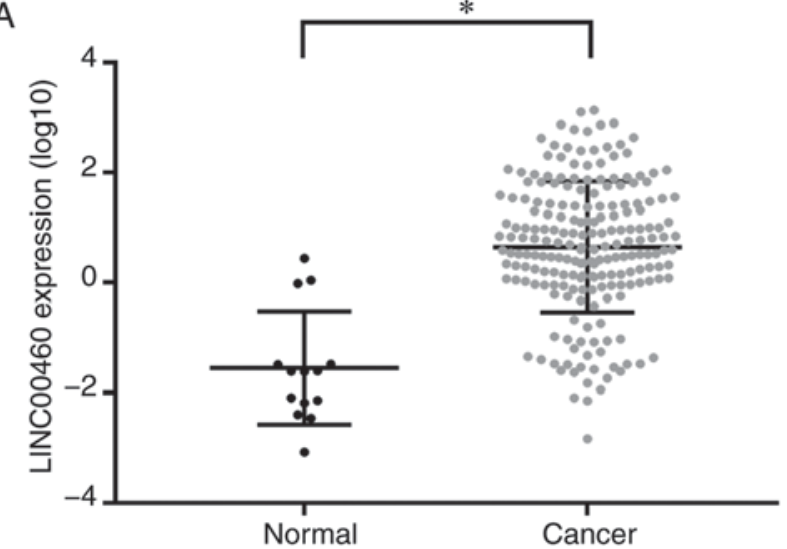

B

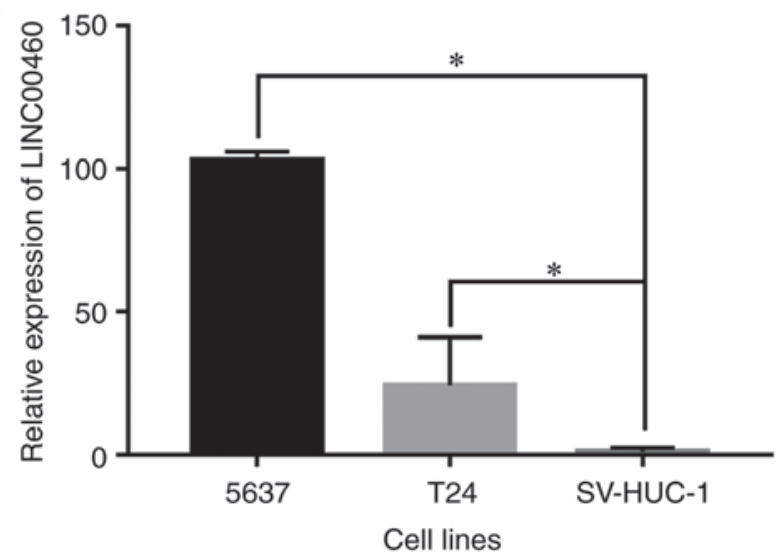

Figure 1. Expression of LINC00460 is upregulated in bladder cancer tissues and cell lines. (A) Relative expression of LINC00460 in bladder cancer tissues and normal controls. (B) Relative expression of LINC00460 in bladder cancer cell lines and a normal bladder epithelial cell line. ${ }^{*} \mathrm{P}<0.05$

assay revealed that the knockdown of LINC00460 decreased the migration distance of cells (Fig. 3D and E). Overall, the results demonstrated that the silencing of LINC00460 may inhibit the proliferation and migration abilities of 5637 and T24 cells.

AR is downregulated in bladder urothelial carcinoma tissues and is negatively correlated with LINC00460 expression. AR expression was analyzed in bladder urothelial carcinoma and adjacent tissues, and its correlation with LINC00460 expression, using TCGA data. AR was significantly downregulated in bladder urothelial carcinoma tissues compared with the normal tissues $(\mathrm{P}<0.05$; Fig. 4A) and was negatively correlated with the expression of LINC00460 ( $\mathrm{P}<0.0001$; $r=-0.4715$; Fig. 4B).

\section{Discussion}

The majority of cases of bladder cancer are non-muscle invasive at the time of initial diagnosis, and the initial treatment options generally focus on tumor resection, and the prevention of recurrence or progression to muscle-invasive disease (25). The 5-year recurrence-free survival rate ranges from $62-89 \%$ for localized muscle invasive bladder cancer following a radical cystectomy (26). However, for patients with metastatic disease, the 5-year survival is decreased, at 5\% (27). The
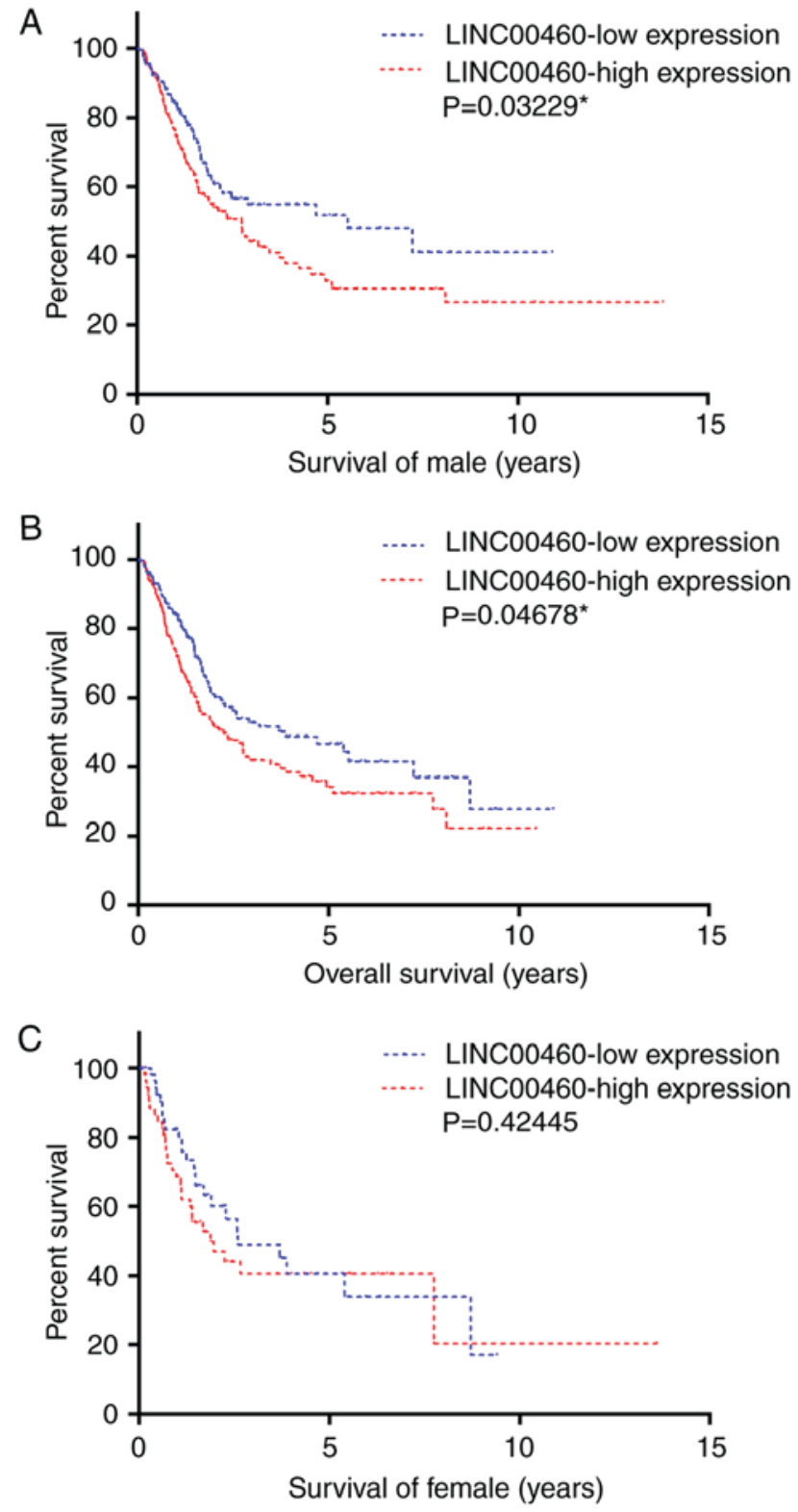

Figure 2. Association between the expression levels of LINC00460 and the overall survival of patients with bladder cancer. A high expression of LINC00460 was associated with poor survival in (A) male patients and (B) the whole patient cohort, but not (C) female patients.

standard drugs used in chemotherapy for the perioperative therapy for MIBC and metastatic disease are cisplatin-based. The median overall survival time with chemotherapy treatment is slightly $>1$ year, with an objective response rate of 40-60\% (28-30). In May 2016, atezolizumab was approved as a second-line therapy for patients with locally advanced or metastatic urothelial carcinoma that had progressed during or following platinum-containing chemotherapy; it became the first available immunotherapeutic antibody to target programmed death-ligand 1. Although targeted therapies have become standard for numerous other malignancies, the number of approved targeted agents in bladder cancer is limited. IncRNAs modulate the expression of genes that are pivotal in the pathways associated with bladder cancer development and progression; for example, the HOX transcript antisense RNA/zinc finger E-box binding homeobox 1 (ZEB1) 
A
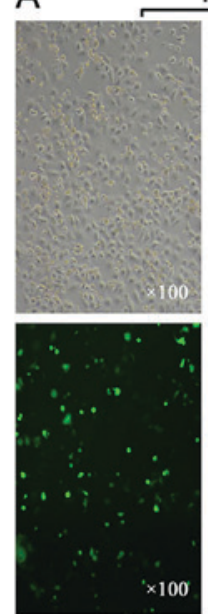

C

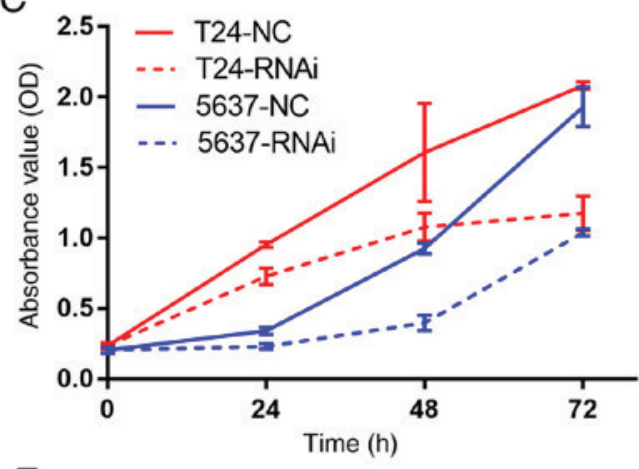

E

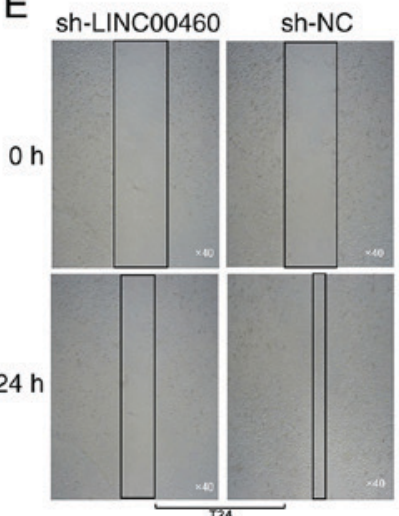

5637

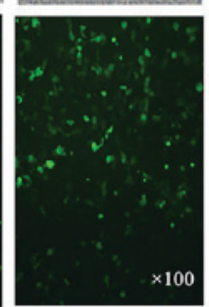

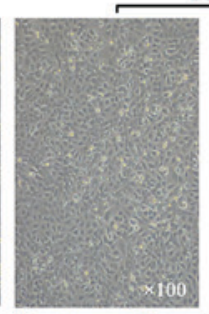

$\mathrm{T} 24$
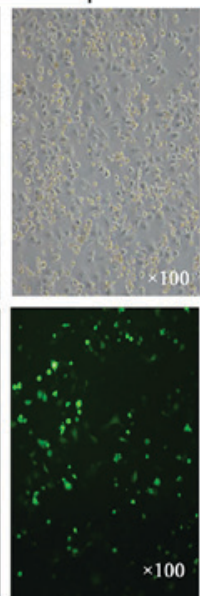

5637
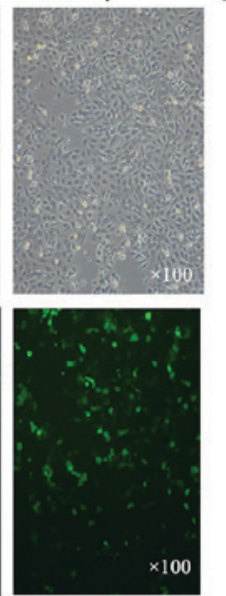

B

D Sh-LINC00460 sh-NC
Cell lines

Negative control

RNAi

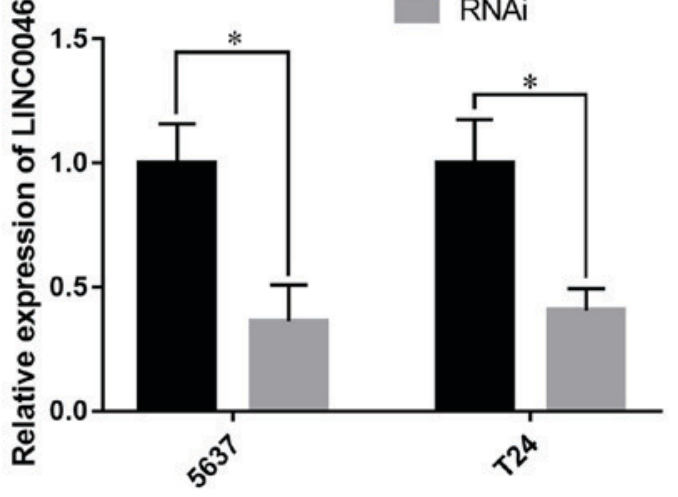

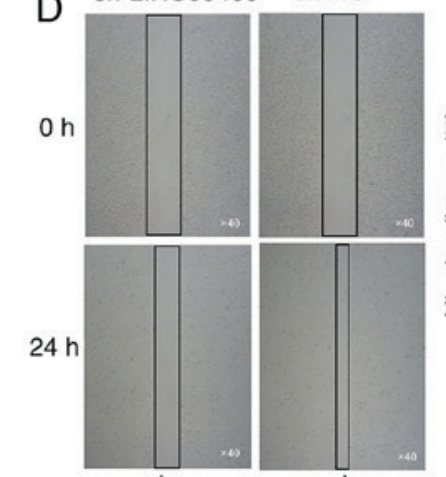

5637
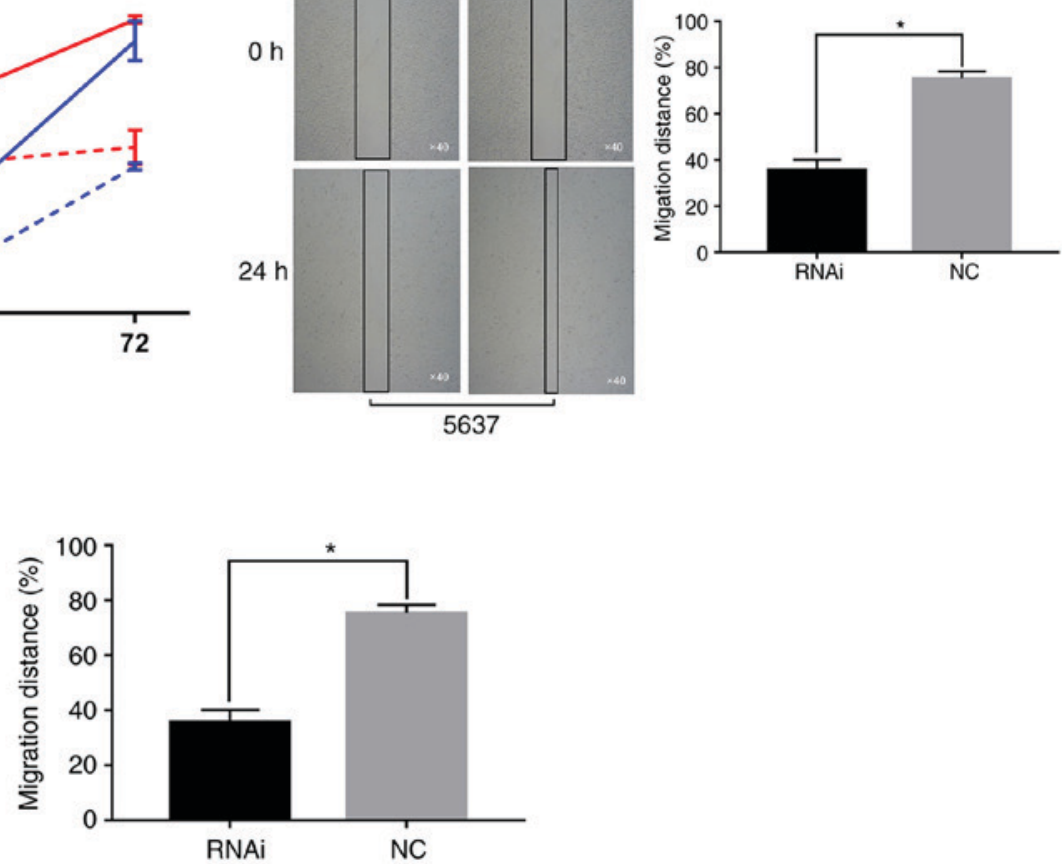

Figure 3. Downregulation of LINC00460 inhibits the proliferation and migration of bladder cancer cells. (A) The green fluorescent protein images indicate the efficiency of transfection. Magnification, x100. (B) Reverse transcription quantitative polymerase chain reaction assays demonstrated that LINC00460 was significantly downregulated following short hairpin RNA transfection. (C) The downregulation of LINC00460 inhibited the proliferation of bladder cancer cells in vitro. The knockdown of LINC00460 decreased the distance of migration in bladder cancer (D) 5637 and (E) T24 cells. Magnification, x40. The experiments were performed in triplicate and data are expressed as the mean \pm standard deviation. ${ }^{~} \mathrm{P}<0.05$. OD, optical density; NC, negative control; RNAi, RNA interference; sh, short hairpin.

interaction affects epithelial-mesenchymal transition in bladder cancer cell lines (31), and metastasis associated lung adenocarcinoma transcript $1 /$ Epithelial cadherin (32) and urothelial cancer associated 1/ZEB1/zinc finger E-box binding homeobox 2 (33) interact to affect the invasion and metastasis of bladder cancer cells. Data from the Cancer Research Network (CRN) database (34) indicated that LINC00460 is upregulated in 14 types of cancer, suggesting that LINC00460 may be a regulator in cancer cell development (35). The role of LINC00460 in bladder cancer was selected for analysis in the present study, as the expression fold change in bladder urothelial carcinoma was the highest among all the types of cancer included in the CRN database.

The present study explored the role of LINC00460 in bladder urothelial carcinoma using TCGA data and cell experiments. The results demonstrated that LINC00460 was upregulated in bladder cancer tissues compared with the corresponding normal controls, while the effect of LINC00460 on the prognosis for bladder urothelial carcinoma was only observed in male patients. Concomitantly, the results also indicated that AR was downregulated in bladder urothelial carcinoma tissues, which was negatively correlated with LINC00460 expression. 
A
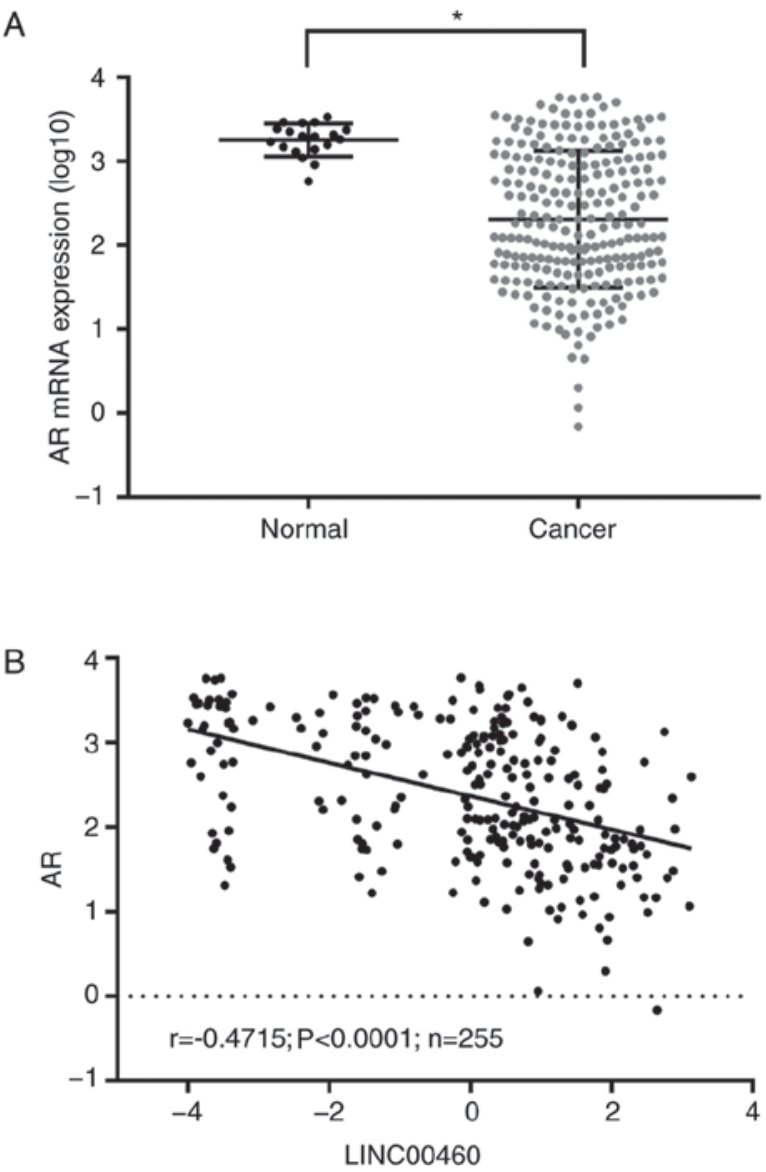

Figure 4. AR is downregulated in bladder urothelial carcinoma tissues and its expression is negatively correlated with the expression of LINC00460. (A) AR mRNA expression was significantly downregulated in bladder urothelial carcinoma tissues. "P<0.05. (B) AR mRNA expression was negatively correlated with the expression of LINC00460 ( $\mathrm{r}=-0.43 ; \mathrm{P}<0.0001$; $\mathrm{n}=255$ ). AR, androgen receptor.

Epidemiological and clinical data have suggested that males are 3-4 times more likely to develop bladder urothelial carcinoma compared with females $(16,36)$. Emerging preclinical evidence has indicated the involvement of AR signaling in the development and progression of bladder urothelial carcinoma; androgens, $\beta$-catenin, cluster of differentiation 24 , cyclins, epidermal growth factor receptor/receptor tyrosine-protein kinase erbB-2, ETS domain-containing protein Elk-1, several AR coregulators and orphan receptors have been demonstrated to directly or indirectly modulate several molecules/pathways involved in bladder urothelial carcinoma cell proliferation (19,20,37-40). As a result, we hypothesized that LINC00460 may serve its oncogenic role by regulating the expression of AR.

The effects of LINC00460 on the proliferation and migration of 5637 and T24 cells were determined using gain- and loss-of-function approaches. The data revealed that the downregulation of LINC00460 inhibited the proliferation and migration of 5637 and T24 cells. Zhang et al (15) performed a regulatory network analysis of LINC00460, and the results indicated that LINC00460 was associated with various biological processes, consistent with the results from the present study.

However, the function of LINC00460 was only investigated in bladder urothelial carcinoma cell lines. An in vivo model is required to confirm the results. In addition, the mechanisms underlying the effect of LINC00460 on 5637 and T24 cells are not yet fully characterized. There was a recurring node, double-strand-break repair protein $\operatorname{rad} 21$ homolog, between mRNAs and transcription factors associated with LINC00460, as identified through bioinformatics methods in a study conducted by Zhang et al (15), which was previously demonstrated to affect cell growth in breast cancer (35). The results from the present study suggested that the expression level of AR mRNA was downregulated in bladder urothelial carcinoma tissues and was negatively correlated with LINC00460. LINC00460 functions as a competing endogenous RNA to upregulate interleukin-6 through sponging miR-149-5p in the cytoplasm of nasopharyngeal carcinoma (NPC) cells (13). LINC00460 was distributed in the cytoplasm and nucleus in NPC cells (13). The data from the present study implied that LINC00460 distributed in the nucleus may serve its role by regulating the expression level of AR mRNA. However, the underlying mechanisms require additional investigation.

In summary, the present study demonstrated that LINC00460 has potential as a clinically promising biomarker for bladder urothelial carcinoma. LINC00460 regulated the proliferation and migration of 5637 and T24 cells, and these data may provide novel insights into molecular cancer therapy.

\section{Acknowledgements}

Not applicable.

\section{Funding}

The present study was supported by the Program of Translational Medicine Research on Bladder Cancer: Construction of Translational Medicine Research Center and Collaborative Network in the Area of Bladder Diseases of Liaoning Province (grant no. 2015225009).

\section{Availability of data and materials}

The datasets used and analyzed during the current study are available from the corresponding author on reasonable request.

\section{Authors' contributions}

PW, MH, MW conceived and supervised the study. LW and $\mathrm{XZ}$ performed the experiments. JB and LH conducted the analysis of data from The Cancer Genome Atlas. HH analyzed the experimental data. LW wrote the manuscript. All authors read and approved the final manuscript.

\section{Ethics approval and consent to participate}

Not applicable.

\section{Patient consent for publication}

Not applicable.

\section{Competing interests}

The authors declare that they have no competing interests. 


\section{References}

1. Ferlay J, Soerjomataram I, Ervik M, Dikshit R, Eser S, Mathers C, Rebelo M, Parkin DM, Forman D and Bray F: Cancer incidence and mortality worldwide: sources, methods and major patterns in GLOBOCAN 2012: Int J Cancer 136: E359-86, 2015.

2. van Rhijn BW, Burger M, Lotan Y, Solsona E, Stief CG, Sylvester RJ, Witjes JA and Zlotta AR: Recurrence and progression of disease in non-muscle-invasive bladder cancer: From epidemiology to treatment strategy. Eur Urol 56: 430-442, 2009.

3. Drayton RM and Catto JW: Molecular mechanisms of cisplatin resistance in bladder cancer. Expert Rev Anticancer Ther 12: 271-281, 2012.

4. Fang Y and Fullwood MJ: Roles, functions, and mechanisms of long non-coding RNAs in cancer. Genomics Proteomics Bioinformatics 14: 42-54, 2016.

5. Jacobs BL, Lee CT and Montie JE: Bladder cancer in 2010: How far have we come? CA Cancer J Clin 60: 244-272, 2010.

6. Gibb EA, Brown CJ and Lam WL: The functional role of long non-coding RNA in human carcinomas. Mol Cancer 10: 38, 2011.

7. Olivieri M,Ferro M, Terreri S, Durso M, Romanelli A, Avitabile C, De Cobelli O, Messere A, Bruzzese D, Vannini I, et al: Long non-coding RNA containing ultraconserved genomic region 8 promotes bladder cancer tumorigenesis. Oncotarget 7: 20636-20654, 2016.

8. Zhao F, Lin T, He W, Han J, Zhu D, Hu K, Li W, Zheng Z, Huang J and Xie W: Knockdown of a novel lincRNA AATBC suppresses proliferation and induces apoptosis in bladder cancer. Oncotarget 6: 1064-1078, 2015.

9. Zhu H, Li X, Song Y, Zhang P, Xiao Y and Xing Y: Long non-coding RNA ANRIL is up-regulated in bladder cancer and regulates bladder cancer cell proliferation and apoptosis through the intrinsic pathway. Biochem Biophys Res Commun 467 223-228, 2015 .

10. He A, Liu Y, Chen Z, Li J, Chen M, Liu L, Liao X, Lv Z, Zhan Y, Zhuang C, et al: Over-expression of long noncoding RNA BANCR inhibits malignant phenotypes of human bladder cancer. J Exp Clin Cancer Res 35: 125, 2016.

11. Taft RJ, Pang KC, Mercer TR, Dinger M and Mattick JS: Non-coding RNAs: Regulators of disease. J Pathol 220: 126-139, 2010.

12. Wang ZL, Li B, Piccolo SR, Zhang XQ, Li JH, Zhou H, Yang JH and Qu LH: Integrative analysis reveals clinical phenotypes and oncogenic potentials of long non-coding RNAs across 15 cancer types. Oncotarget 7: 35044-35055, 2016.

13. Kong YG, Cui M, Chen SM, Xu Y, Xu Y and Tao ZZ: LncRNA-LINC00460 facilitates nasopharyngeal carcinoma tumorigenesis through sponging miR-149-5p to up-regulate IL6. Gene 639: 77-84, 2018.

14. Cao W, Liu JN, Liu Z, Wang X, Han ZG, Ji T, Chen WT and Zou X: A three-lncRNA signature derived from the Atlas of ncRNA in cancer (TANRIC) database predicts the survival of patients with head and neck squamous cell carcinoma. Oral Oncol 65: 94-101, 2017.

15. Zhang Y, Tao Y and Liao Q: Long noncoding RNA: A crosslink in biological regulatory network. Brief Bioinform 19: 930-945, 2018.

16. Siegel RL, Miller KD and Jemal A: Cancer statistics, 2016. CA Cancer J Clin 65: 7-30, 2016.

17. Hartge P, Harvey EB, Linehan WM, Silverman DT, Sullivan JW, Hoover RN and Fraumeni JF Jr: Unexplained excess risk of bladder cancer in men. J Natl Cancer Inst 82: 1636-1640, 1990.

18. Hemelt M, Yamamoto H, Cheng KK and Zeegers MP: The effect of smoking on the male excess of bladder cancer: A meta-analysis and geographicalanalyses. Int J Cancer 124: 412-419, 2009.

19. Overdevest JB, Knubel KH, Duex JE, Thomas S, Nitz MD, Harding MA, Smith SC, Frierson HF, Conaway $M$ and Theodorescu D: CD24 expression is important in male urothelial tumorigenesis and metastasis in mice and is androgen regulated. Proc Natl Acad Sci USA 109: E3588-E3596, 2012.

20. Kawahara T, Shareef HK, Aljarah AK, Ide H, Li Y, Kashiwagi E, Netto GJ, Zheng Y and Miyamoto H: ELK1 is up-regulated by androgen in bladder cancer cells and promotes tumor progression. Oncotarget 6: 29860-29876, 2015.

21. Ding G, Yu S, Cheng S, Li G and Yu Y: Androgen receptor (AR) promotes male bladder cancer cell proliferation and migration via regulating CD24 and VEGF. Am J Transl Res 8: 578-587, 2016.

22. Robinson MD, McCarthy DJ and Smyth GK: edgeR: A Bioconductor package for differential expression analysis of digital gene expression data. Bioinformatics 26: 139-140, 2010.
23. Papatheodorou I, Fonseca NA, Keays M, Tang YA, Barrera E, Bazant W, Burke M, Füllgrabe A, Fuentes AM, George N et al: Expression Atlas: Gene and protein expression across multiple studies and organisms. Nucleic Acids Res 46 (D1): D246-D251, 2018.

24. Livak KJ and Schmittgen TD: Analysis of relative gene expression data using real-time quantitative PCR and the 2(-Delta C(T)) method. Methods 25: 402-408, 2001

25. Pasin E, Josephson DY, Mitra AP, Cote RJ and Stein JP: Superficial bladder cancer: An update on etiology, molecular development, classification, and natural history. Rev Urol 10: 31-43, 2008.

26. Stein JP, Lieskovsky G, Cote R, Groshen S, Feng AC, Boyd S, Skinner E, Bochner B, Thangathurai D, Mikhail M, et al: Radical cystectomy in the treatment of invasive bladder cancer: Long-term results in 1,054 patients. J Clin Oncol 19: 666-675, 2001.

27. Howlader N, Noone AM, Krapcho M, Garshell J, Miller D, Altekruse SF, Kosary CL, Yu M, Ruhl J, Tatalovich Z, et al (eds): SEER cancer statistics review, 1975-2012, Natl Cancer Inst, 2015.

28. Sternberg CN, de Mulder P, Schornagel JH, Theodore C, Fossa SD, van Oosterom AT, Witjes JA, Spina M, van Groeningen CJ, Duclos B, et al: Seven year update of an EORTC phase III trial of high-dose intensity M-VAC chemotherapy and G-CSF versus classic M-VAC in advanced urothelial tract tumours. Eur J Cancer 42: 50-54, 2006

29. von der Maase H, Sengelov L, Roberts JT, Ricci S, Dogliotti L, Oliver T, Moore MJ, Zimmermann A and Arning M: Long-term survival results of a randomized trial comparing gemcitabine plus cisplatin, with methotrexate, vinblastine, doxorubicin, plus cisplatin in patients with bladder cancer. J Clin Oncol 23: 4602-4608, 2005.

30. Saxman SB, Propert KJ, Einhorn LH, Crawford ED, Tannock I, Raghavan D, Loehrer PJ Sr and Trump D: Long-term follow-up of a phase III intergroup study of cisplatin alone or in combination with methotrexate, vinblastine, and doxorubicin in patients with metastatic urothelial carcinoma: A cooperative group study. J Clin Oncol 15: 2564-2569, 1997.

31. Berrondo C, Flax J, Kucherov V, Siebert A, Osinski T, Rosenberg A, Fucile C, Richheimer S and Beckham CJ: Expression of the long non-coding RNA HOTAIR Correlates with disease progression in bladder cancer and is contained in bladder cancer patient urinary exosomes. PLoS One 11: e0147236, 2016.

32. Fan Y, Shen B, Tan M, Mu X, Qin Y, Zhang F and Liu Y: TGF- $\beta$-induced upregulation of malat 1 promotes bladder cancer metastasis by associating with suz12. Clin Cancer Res 20: 1531-1541, 2014.

33. Xue M, Pang H, Li X, Li H, Pan J and Chen W: Long non-coding RNA urothelial cancer-associated 1 promotes bladder cancer cell migration and invasion by way of the hsa-miR-145-ZEB1/2-FSCN1 pathway. Cancer Sci 107: 18-27, 2016.

34. Li JR, Sun CH, Li W, Chao RF, Huang CC, Zhou XJ and Liu CC: Cancer RNA-Seq Nexus: A database of phenotype-specific transcriptome profiling in cancer cells. Nucleic Acids Res 44: D944-D951, 2016.

35. Atienza JM, Roth RB, Rosette C, Smylie KJ, Kammerer S, Rehbock J, Ekblom J and Denissenko MF: Suppression of RAD21 gene expression decreases cell growth and enhances cytotoxicity of etoposide and bleomycin in human breast cancer cells. Mol Cancer Ther 4: 361-368, 2005.

36. Torre LA, Bray F, Siegel RL, Ferlay J, Lortet-Tieulent J and Jemal A: Global cancer statistics, 2012. CA Cancer J Clin 65: 87-108, 2015.

37. Li Y,Zheng Y, Izumi $\mathrm{K}$, Ishiguro $\mathrm{H}, \mathrm{Ye} \mathrm{B}, \mathrm{Li} \mathrm{F}$ and Miyamoto $\mathrm{H}$ : Androgen activates $\beta$-catenin signaling in bladder cancer cells. Endocr Relat Cancer 20: 293-304, 2013.

38. Shiota M, Takeuchi A, Yokomizo A, Kashiwagi E, Tatsugami K, Kuroiwa $\mathrm{K}$ and Naito S: Androgen receptor signaling regulates cell growth and vulnerability to doxorubicin in bladder cancer. J Urol 188: 276-286, 2012.

39. Wu JT, Han BM, Yu SQ, Wang HP and Xia SJ: Androgen receptor is a potential therapeutic target for bladder cancer. Urology 75 : 820-827, 2010

40. Zheng Y, Izumi K, Yao JL and Miyamoto H: Dihydrotestosterone upregulates the expression of epidermal growth factor receptor and ERBB2 in androgen receptor-positive bladder cancer cells. Endocr Relat Cancer 18: 451-464, 2011. 